\title{
Burkholderia cenocepacia-induced delay of acidification and phagolysosomal fusion in cystic fibrosis transmembrane conductance regulator (CFTR)-defective macrophages
}

Correspondence

Miguel A. Valvano

mvalvano@uwo.ca

Received 11 August 2008

Revised 12 September 2008

Accepted 17 September 2008
Julie Lamothe ${ }^{1}$ and Miguel A. Valvano ${ }^{1,2}$

${ }^{1}$ Infectious Diseases Research Group, Department of Microbiology and Immunology, Siebens-
Drake Research Institute, University of Western Ontario, London, ON N6A 5C1, Canada

${ }^{2}$ Department of Medicine, University of Western Ontario, London, ON N6A 5C1, Canada

The Burkholderia cepacia complex (Bcc) is a group of opportunistic bacteria chronically infecting the airways of patients with cystic fibrosis (CF). Several laboratories have shown that Bcc members, in particular $B$. cenocepacia, survive within a membrane-bound vacuole inside phagocytic and epithelial cells. We have previously demonstrated that intracellular $B$. cenocepacia causes a delay in phagosomal maturation, as revealed by impaired acidification and slow accumulation of the late phagolysosomal marker LAMP-1. In this study, we demonstrate that uninfected cystic fibrosis transmembrane conductance regulator (CFTR)-defective macrophages or normal macrophages treated with a CFTR-specific drug inhibitor display normal acidification. However, after ingestion of $B$. cenocepacia, acidification and phagolysosomal fusion of the bacteria-containing vacuoles occur in a lower percentage of CFTR-negative macrophages than CFTR-positive cells, suggesting that loss of CFTR function contributes to enhance bacterial intracellular survival. The CFTR-associated phagosomal maturation defect was absent in macrophages exposed to heat-inactivated $B$. cenocepacia and macrophages infected with a nonCF pathogen such as Salmonella enterica, an intracellular pathogen that once internalized rapidly traffics to acidic compartments that acquire lysosomal markers. These results suggest that not only a defective CFTR but also viable $B$. cenocepacia are required for the altered trafficking phenotype. We conclude that CFTR may play a role in the mechanism of clearance of the intracellular infection, as we have shown before that $B$. cenocepacia cells localized to the lysosome lose cell envelope integrity. Therefore, the prolonged maturation arrest of the vacuoles containing $B$. cenocepacia within $\mathrm{cftr}^{-1-}$ macrophages could be a contributing factor in the persistence of the bacteria within CF patients.

\section{INTRODUCTION}

Cystic fibrosis (CF) is a lethal recessive human genetic disorder caused by mutations in the gene encoding a cAMPregulated chloride channel (cystic fibrosis transmembrane conductance regulator; CFTR) that is predominantly expressed on the apical membrane of epithelial cells (Boucher, 2007; Cheng et al., 1990; Riordan et al., 1989). The airways of CF patients become chronically infected with a restricted subset of opportunistic soil bacteria belonging to the Pseudomonas and Burkholderia genera (Govan et al., 1996; Govan \& Deretic, 1996). Chronic infection leads to

Abbreviations: Bcc, Burkholderia cepacia complex; BcCV, Burkholderia cenocepacia-containing vacuole; $\mathrm{CF}$, cystic fibrosis; CFTR, cystic fibrosis transmembrane conductance regulator; eGFP, enhanced green-fluorescent protein; mRFP1, monomeric red-fluorescent protein 1; TMRdextran, tetramethylrhodamine-dextran. progressive airway tissue destruction, pulmonary dysfunction, and death. Burkholderia cepacia complex (Bcc) strains have become important human opportunistic pathogens (Coenye \& Vandamme, 2003; Mahenthiralingam et al., 2005), particularly in young adult patients with CF. B. cenocepacia is one of the most prevalent and pervasive $\mathrm{Bcc}$ species in Canada and other parts of the world (Speert et al., 2002). Bcc and non-Bcc species of Burkholderia are characterized by their metabolic diversity and ability to adapt to varying environmental conditions, including nutrient limitation, antibiotics, antimicrobial peptides and toxic compounds (Coenye \& Vandamme, 2003).

Survival and persistence within host cells and tissues are believed to play a key role in the adaptation of Burkholderia species to multiple niches, including roots and other plant tissues, free-living cells in the environment, and humans. 
Indeed, Burkholderia species can be found as obligate intracellular endosymbionts in pathogenic fungi (PartidaMartinez \& Hertweck, 2005), as symbiotic and endophytic bacteria inhabiting leaf galls, roots and other tissues of plants (Balandreau \& Mavingui, 2006; Van Oevelen et al., 2002), and within free-living amoebae (Inglis et al., 2000; Lamothe et al., 2004; Landers et al., 2000; Marolda et al., 1999). Direct evidence of ex vivo intracellular survival of Bcc isolates in mammalian cells has been demonstrated in the human respiratory epithelial cell line A549 and in cultures of primary lung epithelial cells (Burns et al., 1996; Keig et al., 2001, 2002; Sajjan et al., 2006). Although it is unclear whether intracellular survival in epithelial cells is relevant in vivo, the capacity of Bcc strains to penetrate epithelial cells correlates with mouse infectivity (Chiu et al., 2001; Cieri et al., 2002). Furthermore, Burkholderia pseudomallei (Jones et al., 1996) and Burkholderia mallei (Ribot \& Ulrich, 2006) survive and replicate in macrophage cell lines, unlike Bcc strains, which survive with minimal or no replication (Lamothe et al., 2007; Martin \& Mohr, 2000; Saini et al., 1999). These differences may be due to the ability of $B$. mallei and $B$. pseudomallei to escape from the phagocytic vacuole shortly after phagocytosis and replicate in the cytosol, a process that requires bacterial effectors secreted by type III secretion systems (Stevens et al., 2002, 2003). In contrast, phagocytized Bcc strains reside in bacteria-containing vacuoles (BcCVs) that at least for the initial hours postinfection do not acidify and avoid fusion with lysosomes by a type III-independent mechanism (Lamothe et al., 2007). The bacterial determinants and the host cell targets involved in the mechanism underlying Bcc-mediated altered phagosome maturation still remain unknown.

The ability of Bcc strains to colonize and infect the mucosal airways of CF patients is not well understood. Recent work has suggested that phagosomes of CFTR-defective alveolar macrophages and neutrophils exhibit a constitutive acidification delay that could contribute to alveolar macrophage dysfunction, presumably facilitating infection by CF-related pathogens such as Pseudomonas aeruginosa (Di et al., 2006). However, an independent study using fluorescence ratio imaging to measure the endosomal $\mathrm{pH}$ against an internal standard unequivocally demonstrated that phagolysosomal acidification in alveolar macrophages is CFTR-independent (Haggie \& Verkman, 2007), although these investigators did not examine directly the ability of CFTR macrophages to clear an intracellular infection. In agreement with the latter study, we demonstrate here that uninfected CFTR-defective macrophages or normal macrophages treated with a CFTRspecific inhibitor display normal acidification. However, following infection with B. cenocepacia, BcCVs from CFTRdefective macrophages exhibit a more pronounced delay in acidification and phagolysosomal fusion than that observed with normal macrophages. The CFTR-associated phagosomal maturation defect was absent in macrophages exposed to heat-inactivated B. cenocepacia and macrophages infected with a non-CF pathogen such as Salmonella enterica, a bacterium that once it becomes intracellular rapidly traffics to acidic compartments that acquire lysosomal markers. We conclude that CFTR somehow contributes to the clearance of the intracellular infection and further maturation of the $\mathrm{BcCVs}$, but that this process is specific for B. cenocepacia and perhaps other pathogens that chronically infect the airways of CF patients.

\section{METHODS}

Reagents and antibodies. Dulbecco's modified Eagle medium (DMEM) and fetal bovine serum (FBS) were purchased form Wisent. Dextran tetramethylrhodamine, dextran Oregon green 514 and dextran FITC (molecular mass $10000 \mathrm{Da}$ ) were from Molecular Probes. The primary monoclonal rat anti-mouse LAMP-1 (ID4B) antibody was from the Developmental Studies Hybridoma Bank. Polyclonal goat anti-mouse EEA1 (IgG) was from Santa Cruz Biotechnology. The secondary Alexa 488-conjugated chicken antirat and chicken anti-goat antibodies were purchased from Molecular Probes. Concanamycin A and $\mathrm{CFTR}_{\text {inh }}-172$ were from Sigma.

Bacterial strains, macrophages and culture conditions. $B$. cenocepacia J2315 is a prototypic strain of the highly transmissible ET12 clone (Govan et al., 1993; Johnson et al., 1994). S. enterica serovar Typhimurium LT2 was from our culture collection. Bacteria were grown at $37{ }^{\circ} \mathrm{C}$ in Luria-Bertani (LB) medium with agitation. B. cenocepacia carrying the arabinose-inducible plasmid pMLBAD-eGFP (Lefebre \& Valvano, 2002), which expresses the enhanced green-fluorescent protein (eGFP), was grown with $2 \%(\mathrm{w} / \mathrm{v})$ arabinose and a final concentration of $100 \mu \mathrm{g}$ trimethoprim $\mathrm{ml}^{-1}$. B. cenocepacia carrying pJR1 (Lamothe et al., 2007), which expresses the monomeric red-fluorescent protein 1 (mRFP1), was grown in the presence of $100 \mu \mathrm{g}$ trimethoprim $\mathrm{ml}^{-1}$, and S. enterica carrying pRSETb-mRFP1 (Campbell et al., 2002) was grown in the presence of $100 \mu \mathrm{g}$ ampicillin $\mathrm{ml}^{-1}$. RAW 264.7 macrophages were derived from BALB/c mice (TIB-71, ATCC). The murine $c f t r^{-1-}$ and $c f t r^{+/+}$macrophage cell lines were obtained from Dr D. Radzioch, McGill University. Bone marrow-derived macrophages from the CF-neo ablation were generated by stable transfection of the retroviral recombinant $\mathrm{J} 2$ construct expressing part of the $\mathrm{v}$-raf and $\mathrm{v}$ $m y c$ genes, as described previously (Blasi et al., 1989). The cell lines were derived from the bone marrow of littermate control mice (born from the same carrier of the CF-neo ablation vector parents). The cftr gene knockout mice and their littermate $c f t r$ parental controls were on the same C57BL/6 genetic background. The ablation of the $\mathrm{cftr}$ gene in the $c f t r^{-1-}$ macrophages was confirmed in our laboratory by PCR amplification of an internal fragment of the murine cftr gene exon 10 using primers CFEx10NT (5'-GGAATTATTAAGCACAGTGGAAGAG$\left.3^{\prime}\right)$ and CFEx10CT (5'-GCTTTACTGGAGAAAGTAAGCTACTAG-3'). Cells were maintained in DMEM with $10 \% \mathrm{FBS}$ and grown at $37{ }^{\circ} \mathrm{C}$ in a humidified atmosphere under $5 \%$ carbon dioxide.

Macrophage infection assays. Bacterial strains were washed twice and resuspended in DMEM $10 \%$ FBS. Heat-inactivated bacteria were obtained by incubation at $60{ }^{\circ} \mathrm{C}$ for $25 \mathrm{~min}$ prior to infection. Under these conditions we have noticed $100 \%$ bacterial cell death upon plating in Luria broth medium. Bacteria were added to macrophage cells grown on glass coverslips at the m.o.i. indicated in each experiment, centrifuged for $1 \mathrm{~min}$ at $300 \mathrm{~g}$ and incubated at $37{ }^{\circ} \mathrm{C}$ with $5 \%$ carbon dioxide. Infected macrophages were washed three times with $1 \times$ PBS (Wisent) and observed under a fluorescence microscope or reincubated at $37{ }^{\circ} \mathrm{C}$ under $5 \%$ carbon dioxide in DMEM containing $10 \%$ FBS. In a previous study (Lamothe et al., 2007), we have investigated the internalization of B. cenocepacia strain J2315 in the mouse macrophage-like cell line RAW 264.7, and determined that an m.o.i. of 30-50 allows us to find a reasonable proportion of infected macrophages with a sufficient number of 
intracellular bacteria for analysis. Therefore, unless otherwise indicated, all the experiments in this study were performed using an m.o.i. of 30 .

Live fluorescence microscopy. For lysosome labelling, macrophages were incubated at $37{ }^{\circ} \mathrm{C}$ with $5 \%$ carbon dioxide in the presence of $250 \mu \mathrm{g} \mathrm{ml}{ }^{-1}$ tetramethylrhodamine-dextran (TMR-dextran) for $2 \mathrm{~h}$ and chased for $1 \mathrm{~h}$ in DMEM with $10 \%$ FBS. Labelling of the endosomal pathway was done by incubating macrophages with $B$. cenocepacia $\mathrm{J} 2315$ for $2 \mathrm{~h}$, followed by a $1 \mathrm{~h}$ chase to allow maturation of the BcCVs, and a final incubation with $250 \mu \mathrm{g}$ TMR-dextran $\mathrm{ml}^{-1}$ for an additional $2 \mathrm{~h}$. External TMR-dextran was removed by serial washes with PBS and the macrophages were immediately visualized. Images were acquired using an Axioscope 2 (Carl Zeiss) microscope with a $\times 100$ oil immersion objective coupled to a Qimaging (Burnaby) cooled charged-coupled device camera.

Immunofluorescence and confocal microscopy. Cells were fixed in $4 \%$ paraformaldehyde $(\mathrm{v} / \mathrm{v})$ for $30 \mathrm{~min}$ at room temperature and incubated for $10 \mathrm{~min}$ with $100 \mathrm{mM}$ glycine in $1 \times$ PBS. Cells were then permeabilized with $0 \cdot 1 \%$ Triton X-100 (v/v) and blocked with $5 \%$ milk powder $(\mathrm{w} / \mathrm{v})$ for $1 \mathrm{~h}$ at room temperature. Permeabilized cells were incubated with primary antibodies, followed by secondary antibodies in $5 \%$ milk powder for $1 \mathrm{~h}$ each at room temperature. Coverslips were mounted onto glass slides using fluorescent mounting medium (DakoCytomation). All secondary antibodies were used at a dilution of $1: 1000$, rat anti-LAMP-1 at $1: 50$ and goat anti-EEA1 at $1: 100$. Confocal microscopy was performed using a Zeiss LSM 510 laser scanning confocal microscope and a $\times 100$ oil immersion objective. In each experiment, the colocalization of LAMP-1 and the BcCVs was determined by examining 21 fields at $\times 100$ and counting the number of BcCVs surrounded by a ring-like structure per macrophage cell.

Analysis of endosomal pH. Macrophages were grown on coverslips in DMEM with $10 \%$ FBS to a confluence of $60 \%$. Monolayers were then incubated simultaneously with $250 \mu \mathrm{g}$ TMR-dextran $\mathrm{ml}^{-1}$ and $250 \mu \mathrm{g} \mathrm{ml}^{-1}$ FITC-conjugated dextran for $2 \mathrm{~h}$ or overnight at $37^{\circ} \mathrm{C}$. External dextran was removed by three washes of $2 \mathrm{ml} \mathrm{PBS}$ and macrophages were reincubated for $2 \mathrm{~h}$ in DMEM $10 \%$ FBS to chase dyes to lysosome compartments. In control experiments, macrophages were preincubated for 20 min with $250 \mathrm{nM}$ concanamycin (to inhibit the activity of the vacuolar ATPase; Whyteside et al., 2005) or $10 \mu \mathrm{M}$ $\mathrm{CFTR}_{\text {inh }}-172$, a thiazolidinone compound that specifically inhibits CFTR ion channel activity and has no other known toxic effects for rodent cells in culture (Ma et al., 2002; Taddei et al., 2004). To activate macrophages, monolayers were incubated with $500 \mathrm{ng} \mathrm{ml}^{-1}$ of $B$. cenocepacia J2315 LPS. Pictures were taken using a Zeiss LSM 510 laser scanning confocal microscope with a $\times 100$ oil immersion objective, and laser power remained constant between each experiment. Fluorescence intensities were analysed using LSM Image Examiner software and presented as fluorescence ratios (FITC/TMR).

Statistical analyses. The statistical analysis was done using GraphPad Prism version 4.03 (GraphPad Software) by two-way ANOVA and Student's $t$ test, depending on the experiment. Data were collected by counting the BcCVs per macrophage cell over 21 fields in triplicate.

\section{RESULTS AND DISCUSSION}

\section{CFTR dysfunction does not alter the early maturation of BcCVs}

To characterize the nature of the compartment occupied by intracellular B. cenocepacia in macrophages with altered CFTR function we used three murine macrophage cell lines: RAW 264.7 macrophages, derived from BALB/c mice, and a pair of congenic $c f t r^{+l+}$ and $c f t r^{-1-}$ macrophage cell lines derived from C57BL/6 mice. CFTR function in RAW 264.7 macrophages was pharmacologically abrogated by treatment with $\mathrm{CFTR}_{\mathrm{inh}}-172$. We performed all of these experiments with B. cenocepacia strain J2315, the index case clinical isolate of the ET12 epidemic clone that has been associated with severe lung deterioration in CF patients (Govan et al., 1993). Previously, our laboratory demonstrated that strain J2315 is poorly internalized by RAW 264.7 macrophages. However, m.o.i. values of 30-50 result in a reasonable proportion of infected macrophages and are sufficient for microscopic analysis (Lamothe et al., 2007). Also, in previous work, we have shown that the phagosomes of RAW 264.7 macrophages containing live $B$. cenocepacia J2315 remain functionally connected to the endocytic pathway, as revealed by the continued access of endocytic tracers, such as fluorescent dextran, to the BcCVs (Lamothe et al., 2007). It should be noted that lack of intracellular bacterial replication and the failure to kill extracellular bacteria prevent us from obtaining viable counts in macrophage infection experiments (Lamothe et al., 2007; Saini et al., 1999). Therefore, to examine the effect of the CFTR status on the intracellular behaviour of B. cenocepacia, we conducted single-cell analyses using a variety of markers that correspond to various stages of phagosomal maturation. We first investigated whether BcCVs in macrophages devoid of CFTR function also remain connected with the endocytic pathway. Macrophages were incubated with live B. cenocepacia for $2 \mathrm{~h}$, allowed to mature for $1 \mathrm{~h}$ and then incubated with TMR-dextran (see Methods). BcCVs in both $\mathrm{cftr}^{+/+}$and $c f t r^{-1-}$ macrophages, as well as BcCVs from infected RAW 264.7 macrophages that were pre-incubated with $\mathrm{CFTR}_{\mathrm{inh}^{-}}{ }^{-}$ 172, incorporated fluorescent dextran (Fig. 1a). Quantitative analysis demonstrated that on average $75 \%$ of BcCVs accumulated external dextran with no statistically significant differences among the cell lines, irrespective of CFTR function (Fig. 1b). Therefore, the CFTR functional status does not affect the interactions of BcCVs with newly formed endocytic vacuoles. We also demonstrated previously that the EEAl protein, a marker for the sorting vesicles of the endocytic pathway, is transiently recruited within minutes to BcCVs but dissociates from these vacuoles after 30-45 min (Lamothe et al., 2007). At 30 min post-infection, EEA1 colocalized with BcCVs in both $c \mathrm{ftr}^{+/+}$and $\mathrm{cfr}^{-1-}$ macrophages (Fig. 2), but as expected, this marker disappeared at later incubation times. Together, these experiments demonstrate that CFTR dysfunction does not alter the early maturation events of the BcCVs.

\section{A dysfunctional CFTR enhances the $B$. cenocepacia-mediated maturation defect of the BcCVs}

In RAW 264.7 macrophages infected with B. cenocepacia there is a delay of up to $6 \mathrm{~h}$ before the late phagosome/ 


\section{(a)}

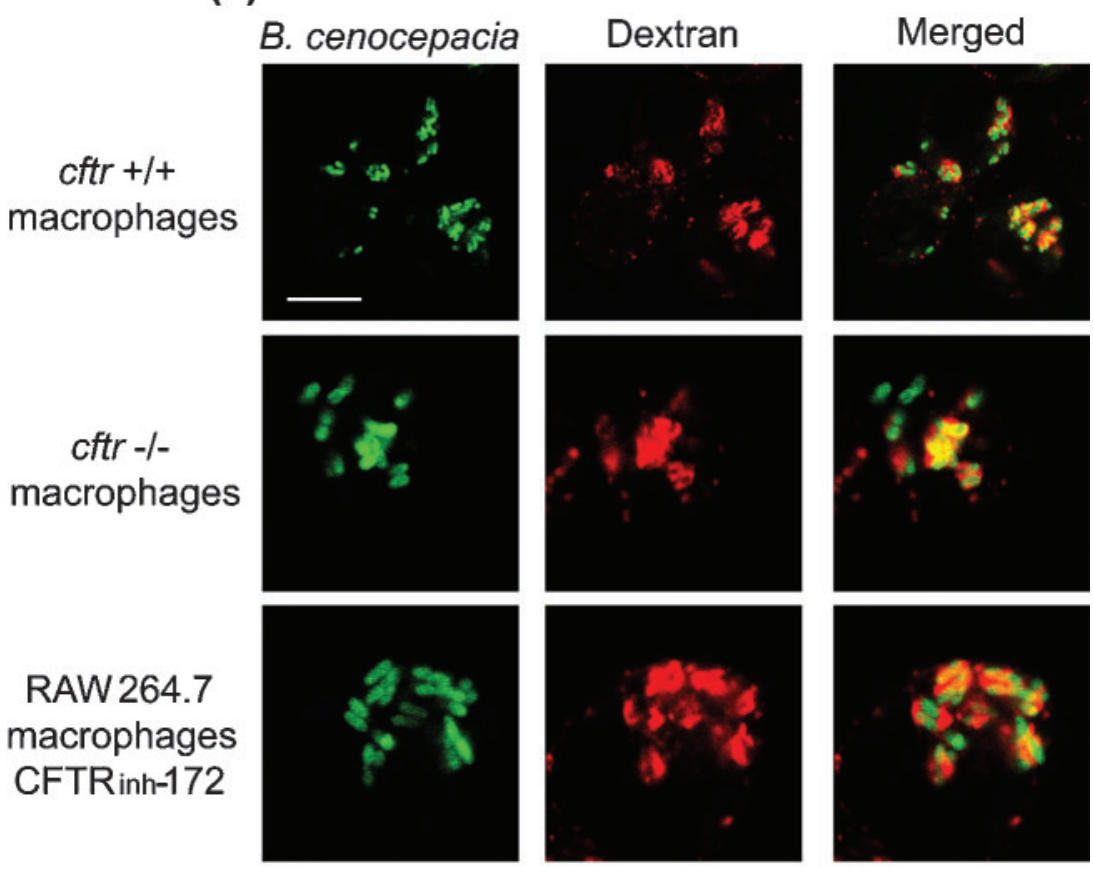

(b)

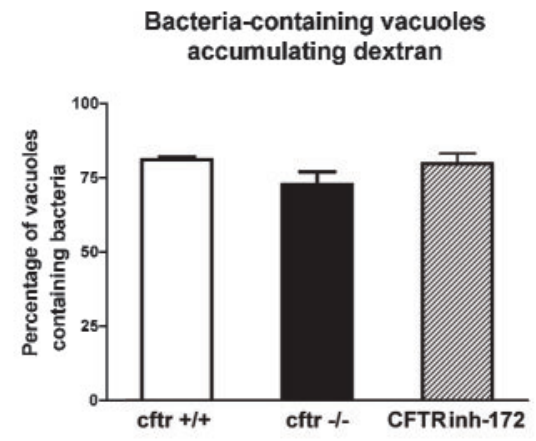

Fig. 1. BcCVs remain functionally associated with incoming endosomes. (a) eGFP fluorescent $B$. cenocepacia J2315, TMR-dextran and merged pictures of $\mathrm{BcCVs}$ from $\mathrm{cftr}^{+/+}$ macrophages (top panels), $\mathrm{cftr}^{-1-}$ macrophages (middle panels) and RAW 264.7 macrophages exposed to $10 \mu \mathrm{M}$ of CFTR $_{\text {-inh }}{ }^{-}$ 172 (bottom panels). All experiments were conduced at an m.o.i. of 30 , which we have previously determined to be optimal conditions for phagocytosis of $B$. cenocepacia (Lamothe et al., 2007). Bar, $11 \mu \mathrm{m}$. (b) Percentage of $\mathrm{BcCV}$ s accumulating external dextran in $\mathrm{cft}^{+/+}$ (white bar), $\mathrm{cftr}^{-1-}$ (black bar) and CFTR $_{\text {-inh }}{ }^{-}$ 172-treated (hatched bar) RAW 264.7 macrophages. The values represent the mean and SD of three experiments in which 21 fields were examined at $\times 100$. Error bars, SEM.

\section{B. cenocepacia}

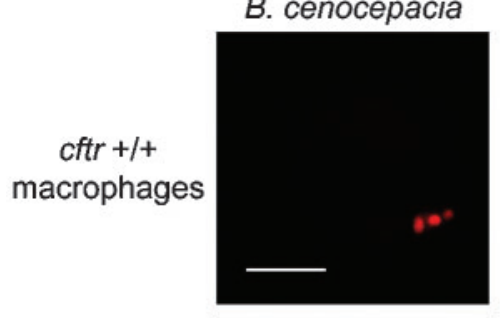

cftr -lmacrophages

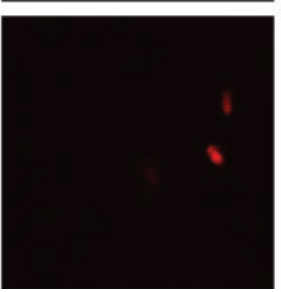

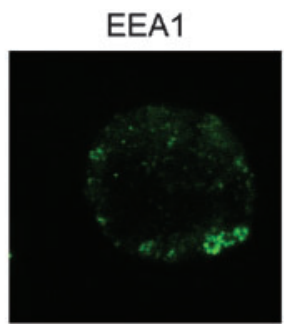
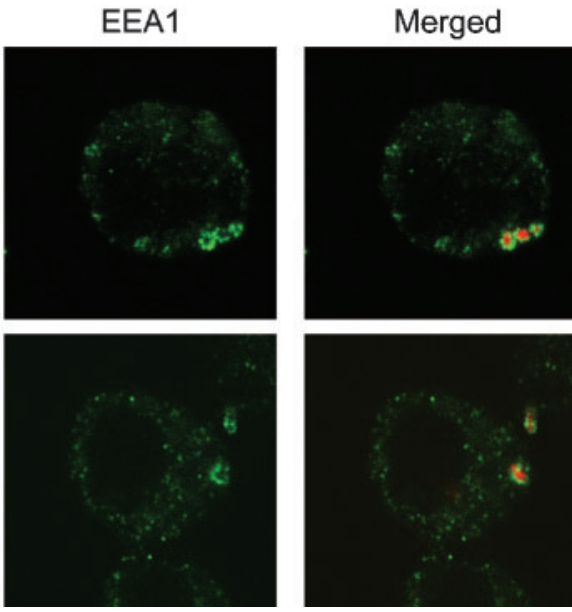

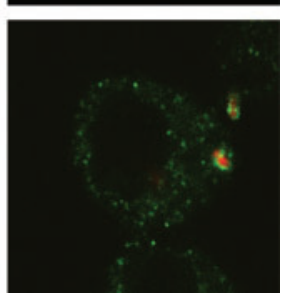

Fig. 2. BcCVs transiently accumulate the early endosomal marker EEA1. cttr $^{+/+}$(top panels) and $\mathrm{cttr}^{-/}$(bottom panels) macrophages were infected with live $B$. cenocepacia J2315 expressing mRFP1 (red fluorescence) at an m.o.i. of 50. Immunolabelling was performed using goat anti-EEA1 and a secondary anti-goat antibody conjugated to AlexaFluor 488 (green fluorescence). Images were captured with a 510 LSM confocal microscope and $\times 100$ objective. Bar, $6 \mu \mathrm{m}$. 
lysosome marker LAMP-1 is clearly detected in the membrane surrounding $\mathrm{BcCVs}$, forming a typical ringlike fluorescent structure (Lamothe et al., 2007). In contrast, at earlier time points, there is a redistribution of the LAMP-1-rich compartment, as demonstrated by images showing clumps of LAMP-1 fluorescence surrounding intracellular bacteria with no typical ring formation (Lamothe et al., 2007). We investigated whether CFTR function is required for this late maturation step by monitoring the temporal accumulation of LAMP-1 into BcCVs of CFTR-defective macrophages. As expected according to our previous study (Lamothe et al., 2007), BcCVs of $\mathrm{CFTR}_{\text {inh }^{-172}}$-treated and -untreated RAW 264.7 cells, as well as BcCVs of $c f t r^{+1+}$ and $c f t r^{-1-}$ macrophages, did not accumulate LAMP-1 between 1 and $4 \mathrm{~h}$ post-infection. However, at $8 \mathrm{~h}$ post-infection, $80 \pm 2 \%$ of $\mathrm{BcCVs}$ from $\mathrm{cftr}^{+/+}$ macrophages acquired LAMP-1, giving rise to typical ring-like structures (Fig. 3a, top-middle panel, arrow- heads, and Fig. 3b). In contrast, rings of LAMP-1-positive material were observed in fewer than $32 \pm 3 \%$ of $\mathrm{BcCVs}$ in $\mathrm{cftr}^{-1-}$ cells $(P<0 \cdot 001$; Fig. 3a, centre panel, and Fig. $3 \mathrm{~b})$. At $8 \mathrm{~h}$ post-infection, several BcCVs in $\mathrm{cftr}^{+/+}$ macrophages also demonstrated a uniform red fluorescent background (Fig. 3a, top-left panel, arrows). We have previously shown that the escape of mRFP1 from the bacterial cytoplasm to the lumen of the BcCVs occurs after phagolysosomal fusion (Lamothe et al., 2007), indicating loss of bacterial cell envelope integrity. This escape of mRFP1 was not observed in BcCVs of infected $c f t r^{-I-}$ macrophages (Fig. 3a, middle-left panel), suggesting that bacteria in these vesicles still have an intact cell envelope. RAW 264.7 macrophages incubated with $\mathrm{CFTR}_{\text {inh }^{-1}}-12$ also did not reveal a significant number of typical rings of LAMP-1 colocalization with BcCVs at $8 \mathrm{~h}$ post-infection (Fig. 3b). However, clumps of LAMP-1positive material were found colocalizing with $\mathrm{BcCVs}$ (Fig. 3a, lower panels). We have previously observed this (a)

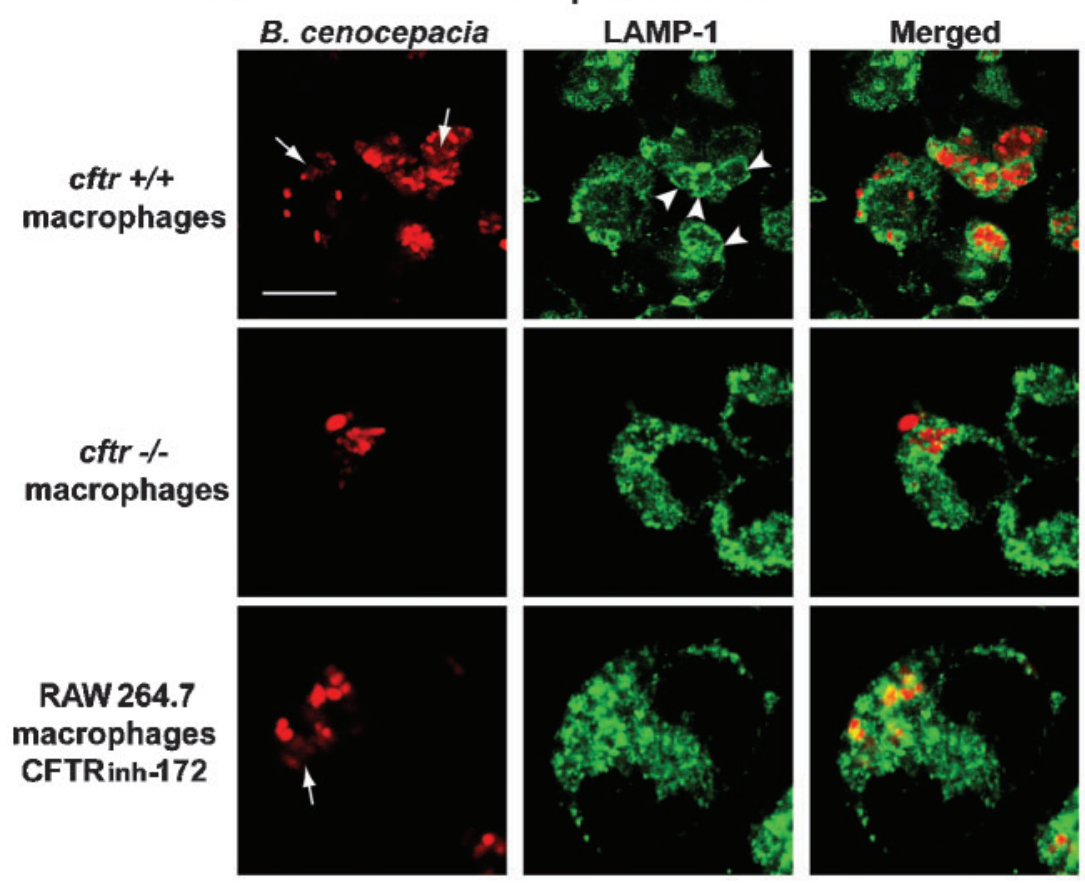

(b)

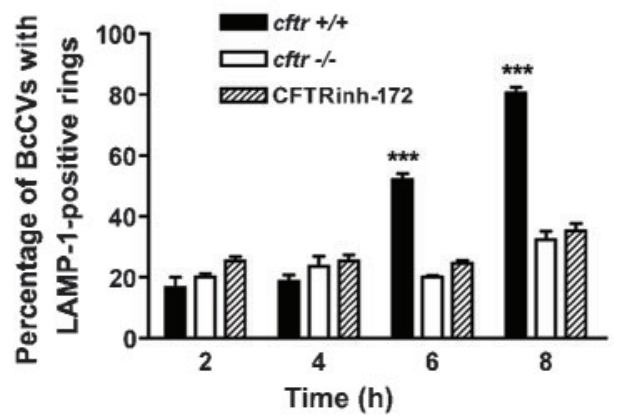

Fig. 3. Colocalization of LAMP-1 to BcCVs. (a) Confocal images of $\mathrm{cttr}^{+/+}, \mathrm{cftr}^{-/-}$and CFTR $_{\text {inh }}$-172-treated RAW 264.7 macrophages infected with $B$. cenocepacia expressing mRFP1 (red fluorescence) at $8 \mathrm{~h}$ post-infection and at an m.o.i. of 30 . Immunolabelling was performed using rat anti-LAMP-1 and a secondary anti-rat antibody conjugated to AlexaFluor 488 (green fluorescence). Arrowheads in the top-middle panel point to typical ring-like structures indicating the colocalization of LAMP-1 with the BcCVs, which is only seen in $\mathrm{cftr}^{+/+}$-infected macrophages. Arrows in the upper- and lower-left panels point to areas of diffuse red fluorescence, which are likely due to the release of mRFP1 content from the bacterial cytoplasm after phagolysosomal fusion (Lamothe et al., 2007). Images were captured with a 510 LSM confocal microscope and a $\times 100$ objective. Bar, $10 \mu \mathrm{m}$. (b) Quantitative analysis of LAMP1 ring formation over the infection time in $\mathrm{ctr}^{+1}$ + (white bar), $\mathrm{cftr}^{-1-}$ (black bar) and CFTR -inh $^{-}$ 172-treated RAW 264.7 (hatched bar) macrophages. Bars represent the mean percentage and SEM from infection experiments in which 21 fields were examined at $\times 100$ for formation of LAMP-1 fluorescent rings around the BcCVs. ${ }^{* \star *}$ The percentage of LAMP-1 accumulation onto $\mathrm{BcCVs}$ of $\mathrm{cftr}^{+/+}$-infected cells at 6 and $8 \mathrm{~h}$ post-infection was significantly higher than in $\mathrm{cftr}^{-/}$cells or in RAW 264.7 macrophages treated with $\mathrm{CFTR}_{\text {-inh }}-172$ $(P<0.001)$. Pair-wise comparisons at other times were non-significant. 
phenomenon in untreated RAW 264.7 macrophages infected with live $B$. cenocepacia just prior to ring formation (Lamothe et al., 2007), but at $1-3 \mathrm{~h}$ postinfection. We also observed in some of the BcCVs the release of mRFP1 to the vacuolar lumen (Fig. 3a, lowerleft panel, arrows), suggesting that phagolysosomal fusion had begun to take place. Therefore, these results suggest that in the presence of $\mathrm{CFTR}_{\mathrm{inh}}-172$ there is also a significant delay in the maturation of the BcCVs, albeit less pronounced than that found with $\mathrm{cftr}^{-1-}$ macrophages.

The pronounced maturation delay observed with $\mathrm{ctr}^{-1-}$ and CFTR $_{\text {inh }}$-172-treated RAW 264.7 macrophages, as judged by the ability of BcCVs to accumulate LAMP-1, was not noticeable when these cells were exposed to heatinactivated B. cenocepacia. Indeed, $\mathrm{BcCV}$ s carrying heatinactivated B. cenocepacia rapidly fused with LAMP-1rich compartments at $30 \mathrm{~min}$ post-infection in the three cell types tested (Fig. 4, see arrowheads). Quantitative analysis revealed that on average $80 \%$ of BcCVs colocalized with LAMP-1-positive material forming ring-like structures in all of the cell types. Therefore, BcCVs containing nonviable B. cenocepacia in $\mathrm{cftr}^{-1-}$ macrophages or in CFTR-inhibited cells trafficked normally into LAMP-1-rich vacuoles, as we have previously found in normal RAW 264.7 macrophages (Lamothe et al., 2007). These results argue that the absence of CFTR expression in $\mathrm{cftr}^{-1-}$ cells or the pharmacological abrogation of CFTR function by $\mathrm{CFTR}_{\text {inh-172 }}$ exaggerates the $B$. cenocepacia-mediated traffic delay of the $\mathrm{BcCVs}$ into the lysosomes.

\section{A dysfunctional CFTR has no impact on the maturation of vacuoles containing $S$. enterica}

To assess whether the absence of a functional CFTR protein has any effect on the trafficking of other intracellular bacteria, we infected $\mathrm{cftr}^{-1-}$ macrophages with $S$. enterica strain LT2. Salmonella is a bona fide intracellular pathogen that promotes its own uptake, and since it has been shown that a high bacterial inoculum may lead to macrophage cell death induced by intracellular Salmonella (Fink \& Cookson, 2007; Monack et al., 1996; Rupper \& Cardelli, 2008), we used a lower m.o.i. for these experiments. It has been reported that the bacteria-containing vacuoles of RAW 264.7 cells infected with $S$. enterica acquire LAMP-1 relatively early after infection (Beuzón et al., 2000; Rathman et al., 1997). At $4 \mathrm{~h}$ post-infection, phagosomes containing live S. enterica from $\mathrm{cftr}^{-1-}$ and RAW 264.7 cells incubated with $\mathrm{CFTR}_{\mathrm{inh}^{-}}$ 172 accumulated LAMP-1 (Fig. 5). These results indicate that the delay in LAMP-1 accumulation is specific to the vacuoles containing live B. cenocepacia and is not a general defect with any viable intracellular bacteria.

\section{Uninfected CFTR-defective macrophages display normal phagolysosomal acidification}

Internalized B. cenocepacia within macrophages reside in poorly acidified vacuoles before fusion with lysosomes (Lamothe et al., 2007). To assess the state of acidification of vacuoles carrying live B. cenocepacia in $\mathrm{cftr}^{-1-}$ macrophages, infected cells were incubated with LysoTracker Red (Invitrogen). This fluorescent probe accumulates preferentially within the most acidic compartments of the cell due to

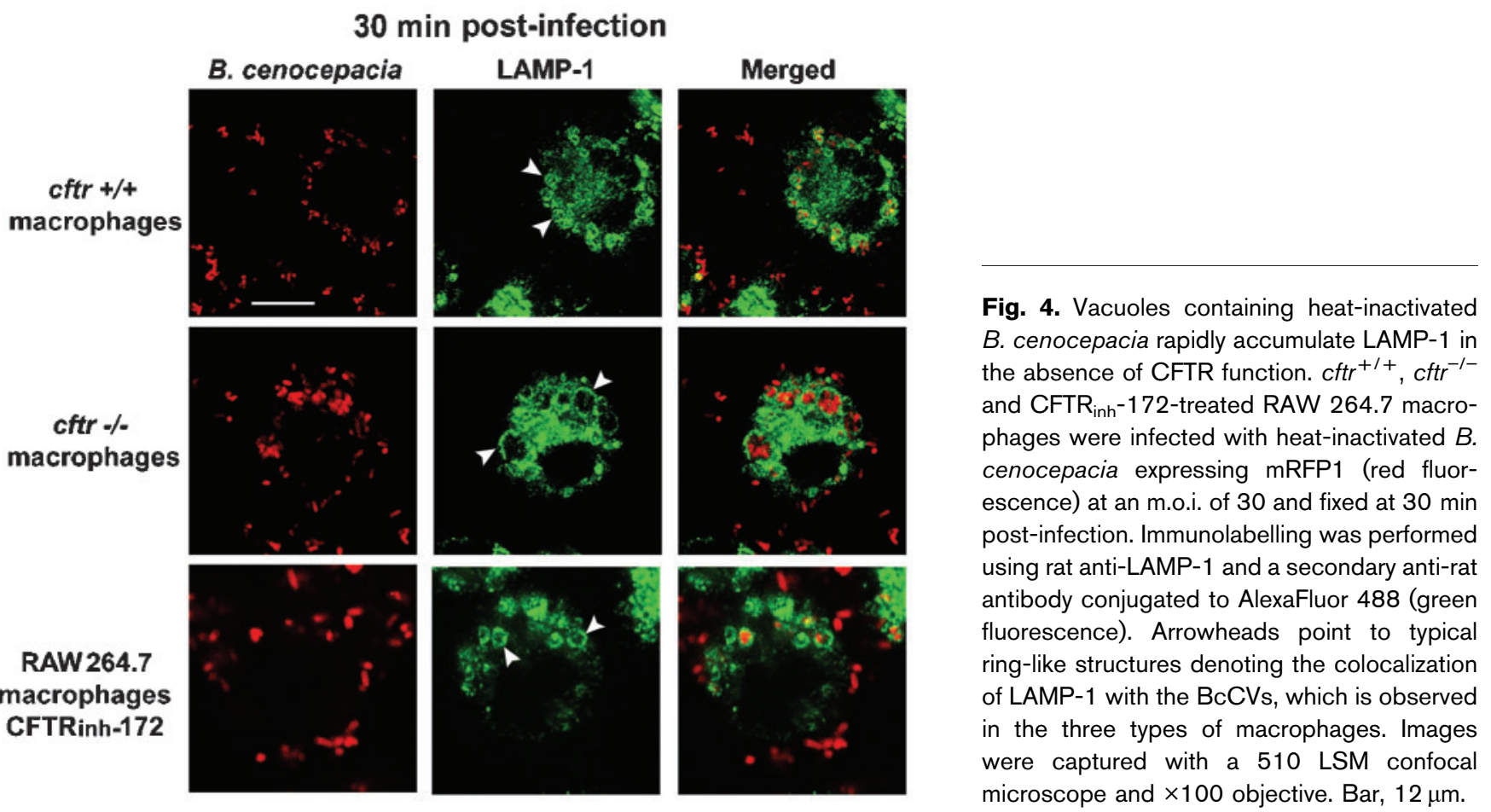




\section{$4 \mathrm{~h}$ post-infection}

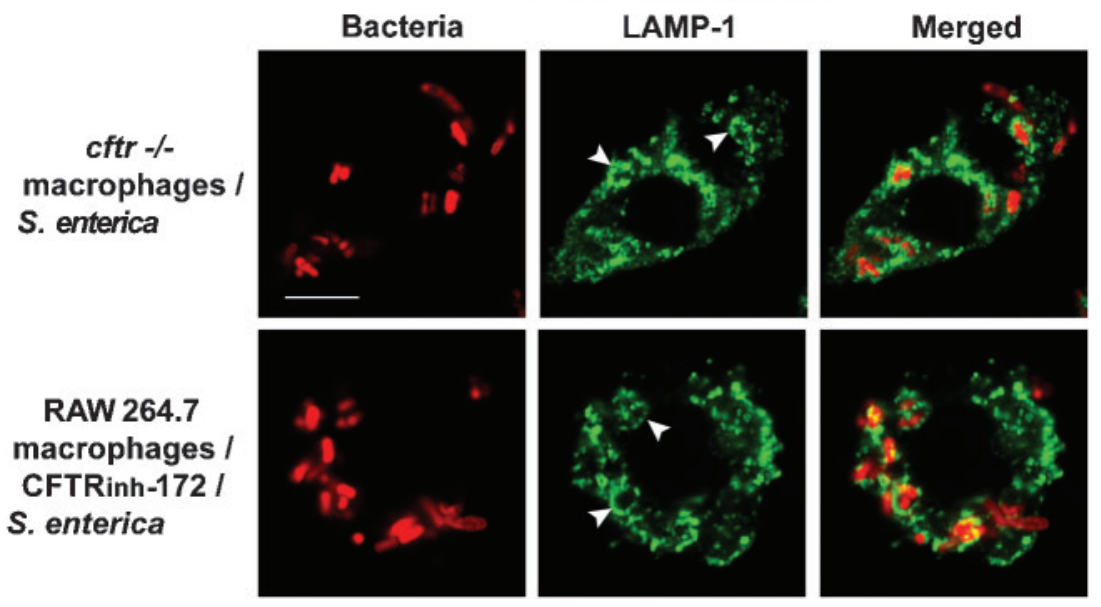

Fig. 5. Colocalization of LAMP-1 with $S$. enterica-containing vacuoles in $\mathrm{cftr}^{-1-}$ and CFTR $_{\text {inh }}$-172-treated RAW 264.7 macrophages. Murine macrophages were infected with live $S$. enterica LT2 expressing mRFP1 (red fluorescence) at an m.o.i. of 10 and fixed at $4 \mathrm{~h}$ post-infection. Immunolabelling was performed using rat anti-LAMP-1 and a secondary anti-rat antibody conjugated with AlexaFluor 488 (green fluorescence). Arrowheads point to typical ring-like structures denoting the colocalization of LAMP-1 with the Salmonella-containing vacuoles. Images were captured with a 510 LSM confocal microscope and $\times 100$ objective. Bar, $6 \mu \mathrm{m}$.

the formation of a membrane-impermeable form upon protonation in low $\mathrm{pH}$ environments (Lemieux et al., 2004). The accumulation of LysoTracker into BcCVs of $c \mathrm{ftr}^{+1+}$ and $c f t r^{-1-}$ macrophages infected with live B. cenocepacia was quantitatively assessed $8 \mathrm{~h}$ after internalization by counting BcCVs in 21 fields in triplicate experiments. At $8 \mathrm{~h}$ post-infection, $23 \pm 4 \%$ of $\mathrm{BcCVs}$ within $c \mathrm{ftr}^{-1-}$ macrophages colocalized with the acidic probe compared with $83 \pm 3 \%$ of $\mathrm{BcCVs}$ within $\mathrm{cftr}^{+/+}$macrophages $(P<0 \cdot 001$; data not shown). These results demonstrate that the vacuoles containing $B$. cenocepacia in $\mathrm{cttr}^{-1-}$ macrophages remain poorly acidified at a time that corresponds to the extended delay in $\mathrm{BcCV}$ maturation. Consistent with poor acidification and the absence of LAMP-1 localization to the BcCVs, vacuoles carrying live B. cenocepacia in $\mathrm{cfr}^{-1-}$ macrophages did not colocalize with dextran-labelled lysosomes (data not shown).

It has recently been suggested that CFTR plays a role in the $\mathrm{pH}$ regulation of alveolar macrophage lysosomes (Di et al., 2006). However, others have observed no $\mathrm{pH}$ variation in organelles of cells that lack CFTR function (Gibson et al., 2000; Haggie \& Verkman, 2007). Since acidification of endosomes is a critical factor for phagosomal maturation (Clague et al., 1994; van Weert et al., 1995) and LAMPs are required for lysosomal fusion (Huynh et al., 2007), we investigated the relationship between CFTR and lysosome acidification in our cell lines. Ratiometric fluorescence analyses between $\mathrm{pH}$-insensitive (TMR-dextran) and $\mathrm{pH}$ sensitive (FITC-dextran) dyes were performed to evaluate acidification of lysosome-like compartments in both resting and activated macrophages. Macrophages from $c f r^{-1-}$ and $c f t r^{+/+}$genetic backgrounds were incubated with $\mathrm{pH}$-sensitive and $\mathrm{pH}$-insensitive fluorescently labelled dextrans (see Methods) and fluorescence ratios (FITC/ TMR) were compared over time. As controls, fluorescence ratios were determined after treatment with $\mathrm{CFTR}_{\text {inh }}-172$ and concanamycin A, a $\mathrm{V}-\mathrm{H}^{+}$ATPase inhibitor (Whyteside et al., 2005). FITC fluorescence in the endosomal compartments from both $c f t r^{-/-}$and $c f t r^{+/+}$ macrophages was quenched, indicating lumenal acidification, but the endosomes remained strongly fluorescent in cells pre-treated with concanamycin A (Fig. 6a). The fluorescence ratios (FITC/TMR) of concanamycin Atreated cells were around 1 , while the fluorescence ratios of $c f t r^{-/-}, c f t r^{+/+}$and RAW 264.7 macrophages treated with the CFTR inhibitor averaged between 0.6 and 0.7 (Fig. 6b), demonstrating no statistically significant differences between the three experiments. It could be argued that one major confounding factor in our experiments is the presence of extracellular bacteria during the entire course of the experiment. However, similar results were also obtained from radiometric analyses using macrophages that were activated by treatment with $500 \mathrm{ng} B$. cenocepacia J2315 LPS (data not shown), a condition that leads to macrophage cell activation by stimulating the synthesis and secretion of tumour necrosis factor- 1 and the oxidative burst (Saini et al., 1999; K. Keith and M. A. Valvano, unpublished results). Together, these data demonstrate that in agreement with an earlier report (Haggie \& Verkman, 2007), uninfected $c f t r^{-1-}$ macrophages or macrophages treated with $\mathrm{CFTR}_{\text {inh }}-172$ do not have an intrinsic defect in their ability to acidify lysosomal compartments. It has been shown elsewhere that the CFTR protein in macrophages and neutrophils can be visualized in the membrane of vacuoles (Di et al., 2006; Painter et al., 2006). Experiments in our laboratory using five different CFTR-specific antibodies, including the one used in an earlier report (Di et al., 2006), did not yield any results. However, CFTR expression was detected by RT-PCR (data not shown). These results support the notion that CFTR is present in these cells at very low levels of expression.

\section{Concluding remarks}

Macrophages are increasingly recognized as having a central role in the maintenance of immunological homeostasis and host defence in the respiratory tract (Holt et al., 


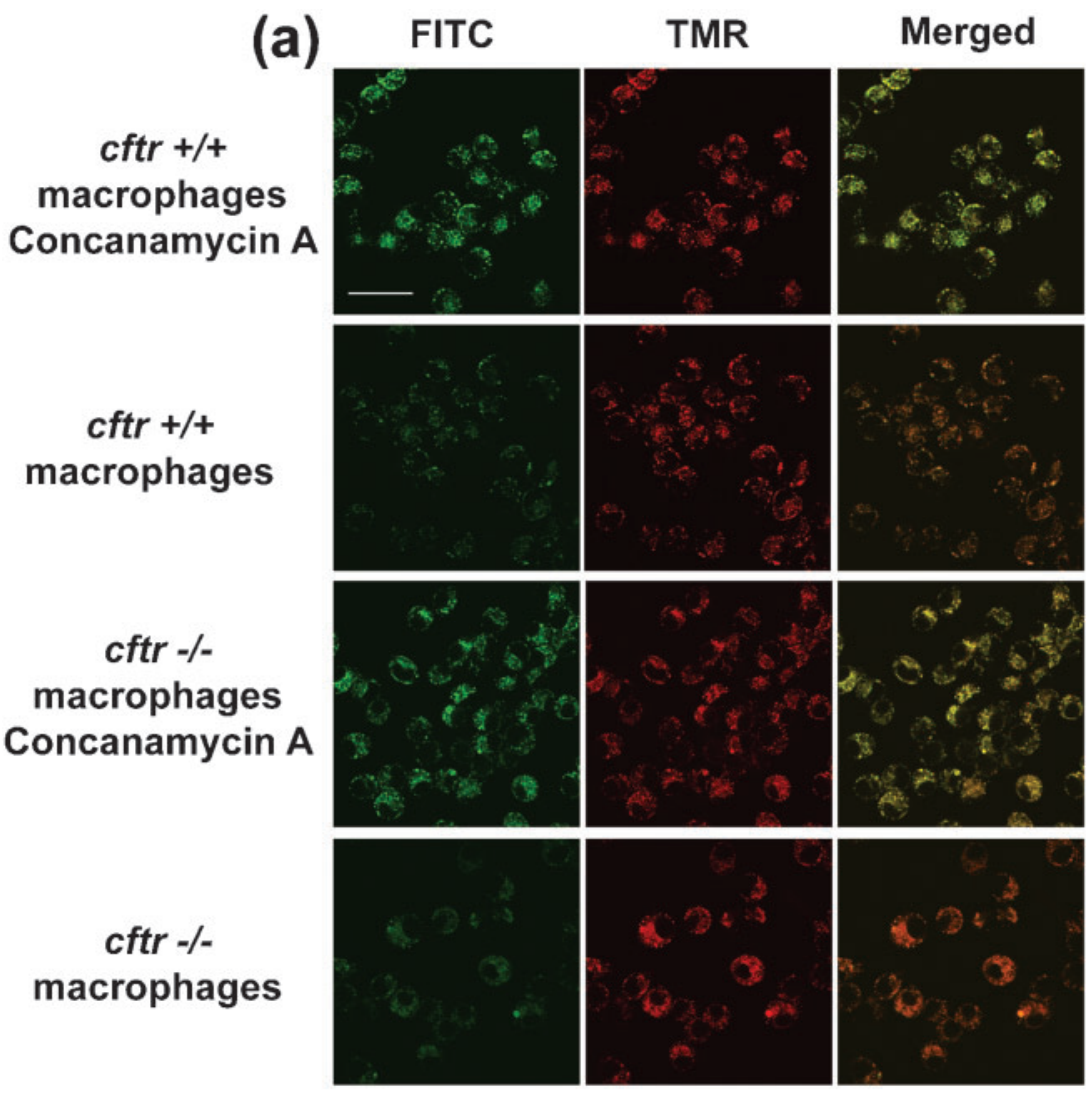

(b) Fluorescence ratio of FITC/TMR
in macrophage lysosomes

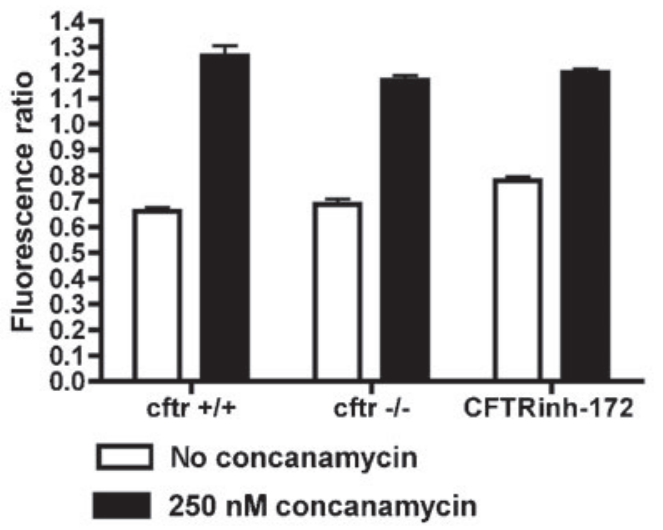

Fig. 6. Determination of endosomal acidification in uninfected $\mathrm{cftr}^{+/+}$and $\mathrm{cftr}^{-1-}$ macrophages. Double labelling of endosomes was conducted by incubating macrophages with $250 \mu \mathrm{g}$ TMR-dextran $\mathrm{ml}^{-1}$ and $250 \mu \mathrm{g}$ FITCdextran $\mathrm{ml}^{-1}$ for $2 \mathrm{~h}$ or overnight, followed by $2 \mathrm{~h}$ post-incubation in dextran-free medium. Concanamycin $(250 \mathrm{nM})$ and CFTR inhibitor $(10 \mu \mathrm{M})$ were added 20 min before dextran labelling and were kept throughout the experiment. Confocal images were captured with a 510 LSM confocal microscope with a $\times 40$ objective and fluorescence ratios were analysed using LSM Image Examiner software. (a) Dextran fluorescence in LPS-activated macrophages. Confocal images were captured and fluorescence ratios analysed using a 510 LSM confocal microscope and $\times 40$ objective. Bar, $40 \mu \mathrm{m}$. (b) Fluorescence ratios (FITC/TMR) of endosomal compartments in resting macrophages not treated (white bars) and treated (black bars) with concanamycin. CFTRinh-172 denotes CFTR $_{\text {inh }}$-172-treated RAW 264.7 macrophages. The values represent the mean and SD of three experiments involving at least 25 randomly picked macrophages per experiment.
2008). Therefore, although the virulence factors responsible for the persistence of $B$. cenocepacia and the bacterial properties associated with tropism for the airways of CF and other immunocompromised patients are still not fully understood, the ability of these bacteria to survive intracellularly may play a role in pathogenesis (Chiu et al., 2001; Valvano et al., 2005, 2006). The purpose of the present study was to determine whether macrophages carrying a mutated $c f t r$ gene or a functionally defective CFTR protein have any alterations in their ability to process B. cenocepacia. Our results demonstrate that in
CFTR-defective macrophages, the $\mathrm{BcCV}$ s exhibit a pronounced delay in phagolysosomal fusion, which is associated with a reduced acidification of the lumen. We also show that this process is specific for live B. cenocepacia, since heat-inactivated bacteria are trafficked normally to the lysosomal compartment upon phagocytosis. Furthermore, the non-CF pathogen S. enterica does not affect the acquisition of the phagolysosomal marker LAMP-1 in CFTR-defective cells. Di et al. (2006) have recently demonstrated that lysosomal compartments within $c \mathrm{ftr}^{-1-}$ alveolar macrophages fail to acidify, leading 
to a bactericidal defect, suggesting that CFTR is involved in the $\mathrm{pH}$ regulation of the phagocytic vacuole. Our results from ratiometric fluorescent intravacuolar $\mathrm{pH}$ measurements do not support the notion that CFTR plays a direct role in the acidification of the phagocytic vacuole, as proposed by $\mathrm{Di}$ et al. (2006), as we did not find a constitutive acidification defect in our $\mathrm{cftr}^{-1-}$ macrophages. This is in agreement with a recent detailed analysis using fluorescence ratio imaging to measure the endosomal $\mathrm{pH}$ against an internal standard, which convincingly demonstrated that phagolysosomal acidification in macrophages is CFTR-independent (Haggie \& Verkman, 2007). It has been reported that in human neutrophils CFTR channel dysfunction affects neutrophil chlorination of phagocytized bacteria (Painter et al., 2006, 2008), raising the possibility that CFTR contributes to bacterial clearance rather than phagosomal acidification. Our experiments with B. cenocepacia-infected CFTR-defective macrophages showing an extended delay in the trafficking of BcCVs to lysosomes do indeed support a role for CFTR in the mechanism of clearance of the intracellular infection, as we have shown before that $B$. cenocepacia localized to the lysosome rapidly loses cell envelope integrity (Lamothe et al., 2007). Furthermore, the prolonged maturation arrest of the vacuoles containing $B$. cenocepacia within $\mathrm{cttr}^{-1-}$ macrophages could explain the persistence of the bacteria within CF patients compared to healthy individuals. Further experiments are currently under way in our laboratory to elucidate the interplay of CFTR and $B$. cenocepacia intracellular survival in macrophages.

\section{ACKNOWLEDGEMENTS}

The authors thank C. Marino, University of Texas Southwestern Medical Center, and D. Radzioch, McGill University, for providing CFTR-specific antibodies and the $c \mathrm{ftr}^{-1-}$ and $c \mathrm{ftr} \mathrm{r}^{+1+}$ macrophage cell lines, and D. Laird, S. Koval and S. Kim for useful comments. This work was supported by operating grants from the Canadian Cystic Fibrosis Foundation. J. L. was supported by studentships from the Canadian Cystic Fibrosis Foundation and a Canada Graduate Doctoral Award from the Canadian Institutes of Health Research. M. A. V. holds a Canada Research Chair in Infectious Diseases and Microbial Pathogenesis.

\section{REFERENCES}

Balandreau, J. \& Mavingui, P. (2006). Beneficial interactions of Burkholderia spp. with plants. In Burkholderia: Molecular Biology and Genomics, pp. 129-151. Edited by T. Coeyne \& P. Vandamme. New York: Horizon Scientific Press.

Beuzón, C. R., Méresse, S., Unsworth, K. E., Ruiz-Albert, J., Garvis, S., Waterman, S. R., Ryder, T. A., Boucrot, E. \& Holden, D. W. (2000). Salmonella maintains the integrity of its intracellular vacuole through the action of SifA. EMBO J 19, 3235-3249.

Blasi, E., Radzioch, D., Merletti, L. \& Varesio, L. (1989). Generation of macrophage cell line from fresh bone marrow cells with a myc/raf recombinant retrovirus. Cancer Biochem Biophys 10, 303-317.

Boucher, R. C. (2007). Cystic fibrosis: a disease of vulnerability to airway surface dehydration. Trends Mol Med 13, 231-240.
Burns, J. L., Jonas, M., Chi, E. Y., Clark, D. K., Berger, A. \& Griffith, A. (1996). Invasion of respiratory epithelial cells by Burkholderia (Pseudomonas) cepacia. Infect Immun 64, 4054-4059.

Campbell, R. E., Tour, O., Palmer, A. E., Steinbach, P. A., Baird, G. S., Zacharias, D. A. \& Tsien, R. Y. (2002). A monomeric red fluorescent protein. Proc Natl Acad Sci U S A 99, 7877-7882.

Cheng, S. H., Gregory, R. J., Marshall, J., Paul, S., Souza, D. W., White, G. A., O'Riordan, C. R. \& Smith, A. E. (1990). Defective intracellular transport and processing of CFTR is the molecular basis of most cystic fibrosis. Cell 63, 827-834.

Chiu, C. H., Ostry, A. \& Speert, D. P. (2001). Invasion of murine respiratory epithelial cells in vivo by Burkholderia cepacia. J Med Microbiol 50, 594-601.

Cieri, M. V., Mayer-Hamblett, N., Griffith, A. \& Burns, J. L. (2002). Correlation between an in vitro invasion assay and a murine model of Burkholderia cepacia lung infection. Infect Immun 70, 1081-1086.

Clague, M. J., Urbe, S., Aniento, F. \& Gruenberg, J. (1994). Vacuolar ATPase activity is required for endosomal carrier vesicle formation. $J$ Biol Chem 269, 21-24.

Coenye, T. \& Vandamme, P. (2003). Diversity and significance of Burkholderia species occupying diverse ecological niches. Environ Microbiol 5, 719-729.

Di, A., Brown, M. E., Deriy, L. V., Li, C., Szeto, F. L., Chen, Y., Huang, P., Tong, J., Naren, A. P. \& other authors (2006). CFTR regulates phagosome acidification in macrophages and alters bactericidal activity. Nat Cell Biol 8, 933-944.

Fink, S. L. \& Cookson, B. T. (2007). Pyroptosis and host cell death responses during Salmonella infection. Cell Microbiol 9, 2562-2570.

Gibson, G. A., Hill, W. G. \& Weisz, O. A. (2000). Evidence against the acidification hypothesis in cystic fibrosis. Am J Physiol Cell Physiol 279, C1088-C1099.

Govan, J. R. W. \& Deretic, V. (1996). Microbial pathogenesis in cystic fibrosis: mucoid Pseudomonas aeruginosa and Burkholderia cepacia. Microbiol Rev 60, 539-574.

Govan, J. R., Brown, P. H., Maddison, J., Doherty, C. J., Nelson, J. W., Dodd, M., Greening, A. P. \& Webb, A. K. (1993). Evidence for transmission of Pseudomonas cepacia by social contact in cystic fibrosis. Lancet 342, 15-19.

Govan, J. R., Hughes, J. E. \& Vandamme, P. (1996). Burkholderia cepacia: medical, taxonomic and ecological issues. J Med Microbiol 45, 395-407.

Haggie, P. M. \& Verkman, A. S. (2007). Cystic fibrosis transmembrane conductance regulator-independent phagosomal acidification in macrophages. J Biol Chem 282, 31422-31428.

Holt, P. G., Strickland, D. H., Wikstrom, M. E. \& Jahnsen, F. L. (2008). Regulation of immunological homeostasis in the respiratory tract. Nat Rev Immunol 8, 142-152.

Huynh, K. K., Eskelinen, E. L., Scott, C. C., Malevanets, A., Saftig, P. \& Grinstein, S. (2007). LAMP proteins are required for fusion of lysosomes with phagosomes. EMBO J 26, 313-324.

Inglis, T. J., Rigby, P., Robertson, T. A., Dutton, N. S., Henderson, M. \& Chang, B. J. (2000). Interaction between Burkholderia pseudomallei and Acanthamoeba species results in coiling phagocytosis, endamebic bacterial survival, and escape. Infect Immun 68, 1681-1686.

Johnson, W. M., Tyler, S. D. \& Rozee, K. R. (1994). Linkage analysis of geographic and clinical clusters in Pseudomonas cepacia infections by multilocus enzyme electrophoresis and ribotyping. J Clin Microbiol 32, 924-930.

Jones, A. L., Beveridge, T. J. \& Woods, D. E. (1996). Intracellular survival of Burkholderia pseudomallei. Infect Immun 64, 782-790. 
Keig, P. M., Ingham, E. \& Kerr, K. G. (2001). Invasion of human type II pneumocytes by Burkholderia cepacia. Microb Pathog 30, 167-170.

Keig, P. M., Ingham, E., Vandamme, P. A. \& Kerr, K. G. (2002). Differential invasion of respiratory epithelial cells by members of the Burkholderia cepacia complex. Clin Microbiol Infect 8, 47-49.

Lamothe, J., Thyssen, S. \& Valvano, M. A. (2004). Burkholderia cepacia complex isolates survive intracellularly without replication within acidic vacuoles of Acanthamoeba polyphaga. Cell Microbiol 6, $1127-1138$.

Lamothe, J., Huynh, K. K., Grinstein, S. \& Valvano, M. A. (2007). Intracellular survival of Burkholderia cenocepacia in macrophages is associated with a delay in the maturation of bacteria-containing vacuoles. Cell Microbiol 9, 40-53.

Landers, P., Kerr, K. G., Rowbotham, T. J., Tipper, J. L., Keig, P. M., Ingham, E. \& Denton, M. (2000). Survival and growth of Burkholderia cepacia within the free-living amoeba Acanthamoeba polyphaga. Eur J Clin Microbiol Infect Dis 19, 121-123.

Lefebre, M. D. \& Valvano, M. A. (2002). Construction and evaluation of plasmid vectors optimized for constitutive and regulated gene expression in Burkholderia cepacia complex isolates. Appl Environ Microbiol 68, 5956-5964.

Lemieux, B., Percival, M. D. \& Falgueyret, J. P. (2004). Quantitation of the lysosomotropic character of cationic amphiphilic drugs using the fluorescent basic amine Red DND-99. Anal Biochem 327, 247-251.

Ma, T., Thiagarajah, J. R., Yang, H., Sonawane, N. D., Folli, C., Galietta, L. J. \& Verkman, A. S. (2002). Thiazolidinone CFTR inhibitor identified by high-throughput screening blocks cholera toxin-induced intestinal fluid secretion. J Clin Invest 110, 1651-1658.

Mahenthiralingam, E., Urban, T. A. \& Goldberg, J. B. (2005). The multifarious, multireplicon Burkholderia cepacia complex. Nat Rev Microbiol 3, 144-156.

Marolda, C. L., Hauröder, B., John, M. A., Michel, R. \& Valvano, M. A. (1999). Intracellular survival and saprophytic growth of isolates from the Burkholderia cepacia complex in free-living amoebae. Microbiology 145, 1509-1517.

Martin, D. W. \& Mohr, C. D. (2000). Invasion and intracellular survival of Burkholderia cepacia. Infect Immun 68, 24-29.

Monack, D. M., Raupach, B., Hromockyj, A. E. \& Falkow, S. (1996). Salmonella typhimurium invasion induces apoptosis in infected macrophages. Proc Natl Acad Sci U S A 93, 9833-9838.

Painter, R. G., Valentine, V. G., Lanson, N. A., Jr, Leidal, K., Zhang, Q., Lombard, G., Thompson, C., Viswanathan, A., Nauseef, W. M. \& other authors (2006). CFTR expression in human neutrophils and the phagolysosomal chlorination defect in cystic fibrosis. Biochemistry 45, 10260-10269.

Painter, R. G., Bonvillain, R. W., Valentine, V. G., Lombard, G. A., Laplace, S. G., Nauseef, W. M. \& Wang, G. (2008). The role of chloride anion and CFTR in killing of Pseudomonas aeruginosa by normal and CF neutrophils. J Leukoc Biol 83, 1345-1353.

Partida-Martinez, L. P. \& Hertweck, C. (2005). Pathogenic fungus harbours endosymbiotic bacteria for toxin production. Nature 437, 884-888.

Rathman, M., Barker, L. P. \& Falkow, S. (1997). The unique trafficking pattern of Salmonella typhimurium-containing phagosomes in murine macrophages is independent of the mechanism of bacterial entry. Infect Immun 65, 1475-1485.

Ribot, W. J. \& Ulrich, R. L. (2006). The animal pathogen-like type III secretion system is required for the intracellular survival of
Burkholderia mallei within J774.2 macrophages. Infect Immun 74, 4349-4353.

Riordan, J. R., Rommens, J. M., Kerem, B., Alon, N., Rozmahel, R., Grzelczak, Z., Zielenski, J., Lok, S., Plavsic, N. \& other authors (1989). Identification of the cystic fibrosis gene: cloning and characterization of complementary DNA. Science 245, 1066-1073.

Rupper, A. C. \& Cardelli, J. A. (2008). Induction of guanylate binding protein 5 by gamma interferon increases susceptibility to Salmonella enterica serovar Typhimurium-induced pyroptosis in RAW 264.7 cells. Infect Immun 76, 2304-2315.

Saini, L. S., Galsworthy, S. B., John, M. A. \& Valvano, M. A. (1999). Intracellular survival of Burkholderia cepacia complex isolates in the presence of macrophage cell activation. Microbiology 145, 3465-3475.

Sajjan, U. S., Yang, J. H., Hershenson, M. B. \& LiPuma, J. J. (2006). Intracellular trafficking and replication of Burkholderia cenocepacia in human cystic fibrosis airway epithelial cells. Cell Microbiol 8, 14561466.

Speert, D. P., Henry, D., Vandamme, P., Corey, M. \& Mahenthiralingam, E. (2002). Epidemiology of Burkholderia cepacia complex in patients with cystic fibrosis, Canada. Emerg Infect Dis 8, 181-187.

Stevens, M. P., Wood, M. W., Taylor, L. A., Monaghan, P., Hawes, P., Jones, P. W., Wallis, T. S. \& Galyov, E. E. (2002). An Inv/Mxi-Spa-like type III protein secretion system in Burkholderia pseudomallei modulates intracellular behaviour of the pathogen. Mol Microbiol 46, 649-659.

Stevens, M. P., Friebel, A., Taylor, L. A., Wood, M. W., Brown, P. J., Hardt, W. D. \& Galyov, E. E. (2003). A Burkholderia pseudomallei type III secreted protein, BopE, facilitates bacterial invasion of epithelial cells and exhibits guanine nucleotide exchange factor activity. $J$ Bacteriol 185, 4992-4996.

Taddei, A., Folli, C., Zegarra-Moran, O., Fanen, P., Verkman, A. S. \& Galietta, L. J. (2004). Altered channel gating mechanism for CFTR inhibition by a high-affinity thiazolidinone blocker. FEBS Lett 558, $52-56$.

Valvano, M. A., Keith, K. E. \& Cardona, S. T. (2005). Survival and persistence of opportunistic Burkholderia species in host cells. Curr Opin Microbiol 8, 99-105.

Valvano, M. A., Maloney, K. E., Lamothe, J. \& Saldias, S. (2006). Intracellular survival of Burkholderia cepacia complex isolates. In Burkholderia: Molecular Biology and Genomics, pp. 283-300. Edited by T. Coeyne \& P. Vandamme. New York: Horizon Scientific Press.

Van Oevelen, S., De Wachter, R., Vandamme, P., Robbrecht, E. \& Prinsen, E. (2002). Identification of the bacterial endosymbionts in leaf galls of Psychotria (Rubiaceae, angiosperms) and proposal of 'Candidatus Burkholderia kirkii' sp. nov. Int J Syst Evol Microbiol 52, 2023-2027.

van Weert, A. W., Dunn, K. W., Gueze, H. J., Maxfield, F. R. \& Stoorvogel, W. (1995). Transport from late endosomes to lysosomes, but not sorting of integral membrane proteins in endosomes, depends on the vacuolar proton pump. J Cell Biol 130, 821-834.

Whyteside, G., Meek, P. J., Ball, S. K., Dixon, N., Finbow, M. E., Kee, T. P., Findlay, J. B. \& Harrison, M. A. (2005). Concanamycin and indolyl pentadieneamide inhibitors of the vacuolar $\mathrm{H}^{+}$-ATPase bind with high affinity to the purified proteolipid subunit of the membrane domain. Biochemistry 44, 15024-15031.

Edited by: P. Cornelis 